JOURNAL OF

SYMPLECTIC GEOMETRY

Volume 10, Number 2, 225-246, 2012

\title{
SYMPLECTIC REDUCTION OF QUASI-MORPHISMS AND QUASI-STATES
}

\author{
Matthew Strom Borman
}

\begin{abstract}
We prove that quasi-morphisms and quasi-states on a closed rational symplectic manifold descend under symplectic reduction to symplectic hyperplane sections. Along the way we show that quasi-morphisms that arise from spectral invariants are the Calabi homomorphism when restricted to Hamiltonians supported on stably displaceable sets.
\end{abstract}

\section{Introduction and results}

1.1. An overview. In the series of papers [EP03, EP06, EP08, EP09], Entov and Polterovich introduced a way to construct quasi-morphisms on the universal cover of the Hamiltonian group and symplectic quasi-states, when $(M, \omega)$ is a closed symplectic manifold. In order to determine if their construction will work for a specific $\left(M^{2 n}, \omega\right)$, one must compute the quantum homology ring $\mathrm{QH}_{2 n}(M, \omega)$ and determine if it contains a field summand, which in practice can be a nontrivial task. In this paper, we introduce a reduction method that allows one to build quasi-morphisms and symplectic quasi-states from known examples, without requiring further quantum homology calculations. This process is presented formally in Theorems 1.3 and 1.4, which are the main results of this paper.

As an example, suppose that $(M, \omega ; \Sigma)$ forms a subcritical polarization where $\Sigma \subset M$ is a complex hypersurface. If $\zeta: C^{\infty}(M) \rightarrow \mathbb{R}$ is a symplectic quasi-state that vanishes on functions with displaceable support, then our construction produces a symplectic quasi-state $\bar{\zeta}: C^{\infty}(\Sigma) \rightarrow \mathbb{R}$. The main tool of the construction is Biran's decomposition theorem [Bir01], which roughly states that the unit disk normal bundle $E_{\Sigma}$, for the hypersurface $\Sigma \subset M$, is symplectomorphic to $M \backslash \Delta$, where in the subcritical case $\Delta$ is a displaceable set (see Section 1.5 for a precise statement). This allows us to define a map $\Theta: C^{\infty}(\Sigma) \rightarrow C^{\infty}(M)$, which lifts functions $H$ on $\Sigma$ to $E_{\Sigma}$, multiplies them by a function that vanished at the boundary of the 
fibers, and then views the result as a function on $M$ using Biran's symplectomorphism. The reduced symplectic quasi-state $\bar{\zeta}: C^{\infty}(\Sigma) \rightarrow \mathbb{R}$ is the result of pulling $\zeta$ back by the map $\Theta$. The construction for quasi-morphisms proceeds in a similar fashion.

This reduction method produces quasi-morphisms and symplectic quasistates on the Hirzebruch surfaces, which were shown to have quasimorphisms and quasi-states by Ostrover [Ost06], but required quantum homology calculations. These and other examples are discussed in Sections 2.2 and 2.3 .

1.2. The Hamiltonian group. Let $\left(M^{2 n}, \omega\right)$ be a closed symplectic manifold, any smooth function $H \in C^{\infty}(M)$ determines a Hamiltonian vector field $\operatorname{sgrad} H$ on $M$ by $\omega(\operatorname{sgrad} H, \cdot)=-d H$. In this manner any timedependent Hamiltonian $F: M \times[0,1] \rightarrow \mathbb{R}$ gives a Hamiltonian isotopy $\phi_{F}=\left\{f_{t}\right\}_{t \in[0,1]}$, by solving the differential equation $\partial_{t} f_{t}=\left(\operatorname{sgrad} F_{t}\right)_{f_{t}}$ with initial condition $f_{0}=\mathbb{1}$. The collection of all such time one maps $f_{1}$ is the Hamiltonian group $\operatorname{Ham}(M, \omega)$. Seen as a Lie group, its Lie algebra is $\mathcal{H}(M)$, all $H \in C^{\infty}(M)$ that are normalized to have mean zero $\int_{M} H \omega^{n}=0$, and the bracket is the Poisson bracket

$$
\{H, K\}=\omega(\operatorname{sgrad} K, \operatorname{sgrad} H)=d H(\operatorname{sgrad} K) .
$$

Any smooth path in $\operatorname{Ham}(M)$ is a Hamiltonian isotopy, so the space of smooth paths based at the identity $\mathcal{P} \operatorname{Ham}(M)$, can be identified with $\mathcal{P H}(M)$, the space of functions $F: M \times[0,1] \rightarrow \mathbb{R}$ such that $F_{t} \in \mathcal{H}(M)$ at all times. The group structure of time-wise product on $\mathcal{P} \operatorname{Ham}(M)$ carries over to $\mathcal{P} \mathcal{H}(M)$ as $\phi_{F} \phi_{G}=\phi_{F \# G}$ and $\phi_{F}^{-1}=\phi_{\bar{F}}$ where

$$
(F \# G)(x, t)=F(x, t)+G\left(f_{t}^{-1}(x), t\right) \quad \text { and } \quad \bar{F}(x, t)=-F\left(f_{t}(x), t\right) .
$$

The universal cover $\widetilde{\operatorname{Ham}}(M)$ is the path space $\mathcal{P} \operatorname{Ham}(M)$ where elements are considered up to homotopy with fixed endpoints. See [MS98, Pol01] for further preliminaries and note we use the sign conventions of [Pol01].

1.3. Quasi-morphisms on $\widetilde{\operatorname{Ham}}(\boldsymbol{M})$. A homogeneous quasi-morphism on a group $G$ is a function $\mu: G \rightarrow \mathbb{R}$ so that $n \mu(g)=\mu\left(g^{n}\right)$ for all $n \in \mathbb{Z}$ and $g \in G$, and for some $C \geq 0$ :

$$
\left|\mu\left(g_{1} g_{2}\right)-\mu\left(g_{1}\right)-\mu\left(g_{2}\right)\right| \leq C, \quad \text { for all } g_{1}, g_{2} \in G .
$$

The smallest such $C$ is the defect and is denoted $D(\mu)$. Homogeneous quasimorphisms are conjugation invariant and they are genuine homomorphisms when restricted to abelian subgroups. On perfect groups, like $\operatorname{Ham}(M)$ and $\widetilde{\operatorname{Ham}}(M)$, they are the best one can hope for in terms of a map to $\mathbb{R}$. If $\mu$ only satisfies (1.1), then $\mu$ is a quasi-morphism and can be homogenized 
by forming $\check{\mu}(g)=\lim _{n \rightarrow \infty} \frac{\mu\left(g^{n}\right)}{n}$, which will be the unique homogeneous quasi-morphism within a bounded distance from $\mu$. See $[$ Cal09, Kot04] for more information about quasi-morphisms.

In [EP03], Entov and Polterovich introduced a way to build homogeneous quasi-morphisms $\mu: \widetilde{\operatorname{Ham}}(M) \rightarrow \mathbb{R}$ using spectral invariants from Hamiltonian Floer theory. We will briefly recall the construction and refer the reader to $\left[\mathbf{E P 0 3 , ~ E P 0 8 , ~ E P 0 9 ] ~ f o r ~ m o r e ~ d e t a i l s . ~ L e t ~} \mathrm{QH}_{*}(M, \omega)\right.$ be the quantum homology ring for $(M, \omega)$, which is $H_{*}(M ; \mathbb{C}) \otimes_{\mathbb{C}} \Lambda_{\omega}$ where $\Lambda_{\omega}$ is a Novikov ring associated to $(M, \omega)$, and its ring structure is given by a deformation of the normal intersection product using Gromov-Witten invariants. To each element $a \in \mathrm{QH}_{*}(M)$ there is an associated spectral invariant, defined in terms of Hamiltonian Floer theory, which is a functional $c(a, \cdot): C^{\infty}(M \times[0,1]) \rightarrow \mathbb{R}$.

With $c(a, \cdot)$, one can define the functional $\mu(a, \cdot): C^{\infty}(M \times[0,1]) \rightarrow \mathbb{R}$ :

$$
\mu(a, F)=\int_{0}^{1} \int_{M} F(x, t) \omega^{n} d t-\operatorname{Vol}(M) \lim _{k \rightarrow \infty} \frac{c\left(a, F^{\# k}\right)}{k},
$$

which descends to a function

$$
\mu(a, \cdot): \widetilde{\operatorname{Ham}}(M) \rightarrow \mathbb{R} .
$$

If $a \in \mathrm{QH}_{2 n}(M)$ is an idempotent and $a * \mathrm{QH}_{2 n}(M)$ is a field [EP09, Remark 1.23], then $\mu(a, \cdot)$ is a homogeneous quasi-morphism. Recently, Usher [Ush11, Theorem 1.4] and Fukaya, Oh, Ohta, Ono [FOOO11, Theorem 1.2] have independently extended this construction using idempotents in the big quantum homology ring, and the associated spectral invariants. Any quasi-morphism build using spectral invariants we will call a spectral quasi-morphism.

Spectral quasi-morphisms have the following two additional properties, where $\widetilde{\operatorname{Ham}}_{U}(M)$ are all Hamiltonian paths generated by Hamiltonians with support in $U$ (not necessarily normalized):

(1) Calabi property: If $U \subset M$ is open and displaceable, then $\mu$ restricted to $\widetilde{\operatorname{Ham}}_{U}(M)$ is the Calabi homomorphism $\mathrm{Cal}_{U}$ given by

$$
\mathrm{Cal}_{U}\left(\phi_{F}\right)=\int_{0}^{1} \int_{U} F_{t} \omega^{n} d t
$$

(2) Stability property: There is some $B>0$, so that for all $F, G \in \mathcal{P} \mathcal{H}(M)$,

$$
\int_{0}^{1} \min _{M}\left(F_{t}-G_{t}\right) d t \leq \frac{\mu\left(\phi_{G}\right)-\mu\left(\phi_{F}\right)}{B} \leq \int_{0}^{1} \max _{M}\left(F_{t}-G_{t}\right) d t .
$$

In the case of spectral quasi-morphisms $B$ can be taken to be $\operatorname{Vol}(M)$. We will say that a quasi-morphism $\mu$ on $\widetilde{\operatorname{Ham}}(M)$ is stable if it has the stability property. 
A subset $X \subset M$ is displaceable if there is an element $\varphi \in \operatorname{Ham}(M)$ such that $\varphi(X) \cap \bar{X}=\emptyset$. A weaker notion is for $X \times S^{1} \subset M \times T^{*} S^{1}$ to be displaceable, in which case $X$ is stably displaceable. Stable displaceability does not imply displaceability as shown in [EP09, Example 1.30]. For spectral quasi-morphisms, the Calabi property can be strengthen to include stably displaceable sets, which will be proved in Section 2.1 .

Theorem 1.1. Let $U \subset(M, \omega)$ be a stably displaceable open subset, then spectral quasi-morphisms $\mu(a, \cdot): \widetilde{\operatorname{Ham}}(M) \rightarrow \mathbb{R}$ restrict to the Calabi homomorphism $\mathrm{Cal}_{U}$ on $\widetilde{\operatorname{Ham}}_{U}(M)$.

Recently, Shelukhin [She11, Corollary 1] proved that there is a quasimorphism on $\widetilde{\operatorname{Ham}}(M)$ for any closed symplectic manifold, using a construction that is not Floer-theoretic. Theorems 1.3 and 1.4 below cannot be directly applied to Shelukhin's quasi-morphisms since they do not have the Calabi property and they do not induce quasi-states.

1.4. Symplectic quasi-states and quasi-measures. Symplectic quasistates are functionals $\zeta: C^{\infty}(M) \rightarrow \mathbb{R}$ such that for $H, K \in C^{\infty}(M), a \in \mathbb{R}$ :

(1) Quasi-linearity: If $\{H, K\}=0$, then $\zeta(H+a K)=\zeta(H)+a \zeta(K)$.

(2) Monotonicity: If $H \leq K$, then $\zeta(H) \leq \zeta(K)$.

(3) Normalization: $\zeta(1)=1$.

Note that these properties imply that $\zeta$ is Lipschitz with respect to the $C^{0}$-norm, $|\zeta(H)-\zeta(K)| \leq\|H-K\|$.

This notion was introduced by Entov and Polterovich [EP06] and is the symplectic version of Aarnes' notion of a topological quasi-state [Aar91]. These are functionals $\zeta: C(X) \rightarrow \mathbb{R}$, for $X$ a compact space, that are monotonic, normalized, and quasi-linear in the sense that if $F \in C(X)$ and $g, h \in C(\mathbb{R})$, then $\zeta(g \circ F+h \circ F)=\zeta(g \circ F)+\zeta(h \circ F)$. Aarnes proved a Riesz representation theorem for quasi-states showing they correspond to topological quasi-measures, which are additive set functions $\tau: \mathcal{A} \rightarrow[0,1]$ where $\mathcal{A}$ are the subsets of $X$ that are either open or closed. The quasi-state $\zeta$ determines the quasi-measure $\tau_{\zeta}$, where for a closed set $C \subset X$

$$
\tau_{\zeta}(C)=\inf \left\{\zeta(F) \mid F: X \rightarrow[0,1], F \geq \mathbb{1}_{C}\right\} .
$$

Being a symplectic quasi-state is a stronger condition than being a topological quasi-state.

A stable homogeneous quasi-morphism $\mu: \widetilde{\operatorname{Ham}}(M) \rightarrow \mathbb{R}$ induces a symplectic quasi-state $\zeta_{\mu}: C^{\infty}(M) \rightarrow \mathbb{R}$ via

$$
\zeta_{\mu}(H)=\frac{\int_{M} H \omega^{n}-\mu\left(\phi_{H_{n}}\right)}{\operatorname{Vol}(M)}
$$


where $H_{n}=H-\frac{\int_{M} H \omega^{n}}{\operatorname{Vol}(M)}$. Quasi-linearity of $\zeta_{\mu}$ follows from the fact that $\phi_{H}$ and $\phi_{K}$ commute if $\{H, K\}=0$, and quasi-morphisms are homomorphisms on abelian subgroups. The monotonicity of $\zeta_{\mu}$ follows from the stability property for $\mu$.

Symplectic quasi-states formed as in (1.4) are $\operatorname{Ham}(M)$ invariant, since $\mu\left(\phi_{H}\right)=\mu\left(\psi^{-1} \phi_{H} \psi\right)$ by conjugation invariance of quasi-morphisms, and $\psi^{-1} \phi_{H} \psi=\phi_{H \circ \psi}$. If $\mu$ has the Calabi property, then $\zeta_{\mu}$ vanishes on functions with displaceable support and hence the associated quasi-measure $\tau_{\mu}$ will vanish on displaceable sets. Since the symplectic quasi-state $\zeta_{\mu}$ is $\operatorname{Ham}(M)$ invariant, the associated quasi-measure $\tau_{\mu}$ will also be $\operatorname{Ham}(M)$ invariant, which allows it to detect nondisplaceability. For if $\varphi \in \operatorname{Ham}(M)$ displaced $X \subset M$, then $\tau_{\mu}(X) \leq 1 / 2$ since

$$
2 \tau_{\mu}(X)=\tau_{\mu}(X)+\tau_{\mu}(\varphi(X))=\tau_{\mu}(X \cup \varphi(X)) \leq 1 .
$$

Consider now a spectral quasi-morphism $\mu(a, \cdot): \widetilde{\operatorname{Ham}}(M) \rightarrow \mathbb{R}$. The associated symplectic quasi-state $\zeta(a, \cdot): C^{\infty}(M) \rightarrow \mathbb{R}$ is given by

$$
\zeta(a, H)=\lim _{k \rightarrow \infty} \frac{c(a, k H)}{k},
$$

and we can form the associated symplectic quasi-measure $\tau(a, \cdot)$. In $[$ EP09, Definition 1.4], the notion of a closed subset $X \subset M$ being (super)heavy (with respect to an idempotent $a$ ) was introduced. When $a$ gives a spectral quasi-morphism, these two notions agree and are equivalent to $\tau(a, X)=1$ [EP09, Remark 1.23]. In this language they prove [EP09, Theorem 1.4] that if $X$ is stably displaceable, then $\tau(a, X) \neq 1$. This result can be strengthen to the following theorem, which is proven in Section 3.6.

Theorem 1.2. For any subset $V \subset M$, if $V$ is stably displaceable, then $\tau(a, V)=0$.

For a symplectic quasi-state $\zeta: C^{\infty}(M) \rightarrow \mathbb{R}$, consider the inequality

$$
\Pi_{\zeta}(H, K):=|\zeta(H+K)-\zeta(H)-\zeta(K)| \leq C \sqrt{\|\{H, K\}\|} .
$$

In [EPZ07, Theorem 1.4], it is proved that (1.5) holds for symplectic quasistates formed from stable homogeneous quasi-morphisms as in (1.4), where $C$ can be taken to be $\sqrt{2 D(\mu)} / \operatorname{Vol}(M)$. If (1.5) holds for some $C$, let $C(\zeta)$ be the smallest number for which it is satisfied for all $H, K \in C^{\infty}(M)$. Due to a result of Cardin and Viterbo [CV08, Theorem 1.2], which was later generalized by Entov and Polterovich [EP10b, Theorem 1.1] and Buhovsky [Buh10, Theorem 1.1.2], it makes sense to say that two continuous functions $H$ and $K$ Poisson commute if they have smooth $C^{0}$-approximations $H_{n}$ and $K_{n}$ such that $\left\|\left\{H_{n}, K_{n}\right\}\right\| \rightarrow 0$. Therefore (1.5) says that symplectic quasi-states are linear on continuous functions that Poisson commute. The 
inequality (1.5) is the main tool used to lower bound the Poisson bracket invariants introduced by Buhovsky, Entov, Polterovich [BEP12].

\subsection{Polarizations and Biran's decomposition theorem.}

Definition 1.1. A polarization $\mathcal{P}=\left(M^{2 n}, \Omega, J ; \Sigma\right)$ is a closed Kähler manifold with $[\Omega] \in H^{2}(M, \mathbb{Z})$ and a closed connected complex hypersurface $\Sigma \subset M$ such that $[\Sigma] \in H_{2 n-2}(M, \mathbb{Z})$ is Poincaré dual to $k[\Omega] \in H^{2}(M, \mathbb{Z})$. By scaling $\Omega$ we will assume $k=1$ and $\operatorname{Vol}(M, \Omega)=\operatorname{Vol}\left(\Sigma, \Omega_{\Sigma}\right)$.

Given a polarization $\mathcal{P}=(M, \Omega, J ; \Sigma)$, there is a compact isotropic cellular subspace $\Delta_{\mathcal{P}} \subset M$, which is disjoint from $\Sigma$ and is called the skeleton associated to $\mathcal{P}$. An example of a polarization is a linear $\mathbb{C P}^{n-1} \subset \mathbb{C P}^{n}$, where $\mathbb{C P}^{n}$ is given the Fubini-study form $\Omega$ with $\int_{\mathbb{C P}^{1}} \Omega=1$. If $\mathbb{C P}^{n-1}$ is given by $\left\{z_{0}=0\right\}$, then the skeleton $\Delta=\{[1: 0: \cdots: 0]\}$ is a single point.

One can build a symplectic disk bundle $\pi:\left(E_{\Sigma}, \omega_{\text {can }}\right) \rightarrow \Sigma$ using the normal bundle from $\Sigma \subset M$, such that $\left(\Sigma, \omega_{\Sigma}\right)$ symplectically embeds into $\left(E_{\Sigma}, \omega_{\text {can }}\right)$ as the zero section. Biran's decomposition theorem for polarizations [Bir01, Theorem 2.6.A] says there is a canonical symplectomorphism $F_{\mathcal{P}}$ such that the following diagram commutes:

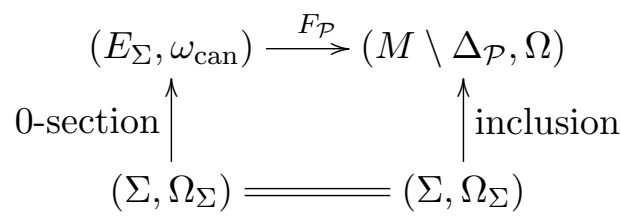

The disk bundle $E_{\Sigma}$ comes with a hermitian metric on the fibers such that each fiber is the open disk of radius one. The radius on the fibers gives a global coordinate function $r$ on $E_{\Sigma}$ and the map $F_{\mathcal{P}}$ is such that any open neighborhood of $\Delta_{\mathcal{P}}$ contains the complement of $F_{\mathcal{P}}\left(\left\{e \in E_{\Sigma} \mid r(e) \leq 1-\epsilon\right\}\right)$ for some small $\epsilon$. For more details see Section 3.1 and [Bir01, Bir06, BC01].

1.6. Symplectic reduction of quasi-morphisms and symplectic quasi-states. We will now describe our procedure for reducing quasimorphisms and symplectic quasi-states from the total space $M$ of a polarization to the hypersurface $\Sigma$, which is the main idea in this paper.

Let $\theta:[0,1] \rightarrow \mathbb{R}$ be a nonnegative function such that $\theta(0)=1$, vanishes in a neighborhood of 1 , and defines a smooth function $\theta(r)$ on $E_{\Sigma}$, where $r$ is the radial coordinate on $E_{\Sigma}$. To $\theta$ we associate a linear, order preserving map

$$
\Theta: C^{\infty}(\Sigma) \rightarrow C^{\infty}(M) \text { given by } \Theta(H)=\left(F_{\mathcal{P}}^{-1}\right)^{*}\left(\theta \cdot \pi^{*} H\right),
$$

where $\pi: E_{\Sigma} \rightarrow \Sigma$ is the projection and $F_{\mathcal{P}}$ is the symplectomorphism in (1.6). When notationally convenient we will consider $\theta$ as the function $\Theta(1)$ on $M$, and observe that the condition that $\theta$ vanishes near 1 ensures that 
$\Theta(H): M \rightarrow \mathbb{R}$ is smooth. If $H \in C^{\infty}(\Sigma)$ has zero mean, then so does $\theta \cdot \pi^{*} H \in C^{\infty}\left(E_{\Sigma}\right)$ (see (3.2)). Therefore we can view $\Theta$ as a linear map

$$
\Theta: \mathcal{P H}(\Sigma) \rightarrow \mathcal{P H}(M)
$$

A nice class of maps are given by $\Theta_{\epsilon}$, where $\theta_{\epsilon}(r):[0,1] \rightarrow \mathbb{R}$ interpolates between $1-r^{2}$ when $r \leq 1-\epsilon$ and zero when $r \geq 1-\frac{\epsilon}{2}$.

Theorem 1.3. Let $\mu: \widetilde{\operatorname{Ham}}(M) \rightarrow \mathbb{R}$ be a homogeneous quasi-morphism and suppose there is an open neighborhood $U$ of $\Delta_{\mathcal{P}}$ so that $\mu$ restricts to the Calabi homomorphism on $\widetilde{\operatorname{Ham}}(M)_{U}$. Then $\mu$ descends to a homogeneous quasi-morphism $\hat{\mu}$, where for any $\epsilon$ sufficiently small:

$$
\hat{\mu}: \widetilde{\operatorname{Ham}}(\Sigma) \rightarrow \mathbb{R} \quad \text { is defined by } \hat{\mu}(\varphi)=\mu\left(\phi_{\Theta_{\epsilon}(F)}\right),
$$

where $F \in \mathcal{P H}(\Sigma)$ generates $\varphi \in \widetilde{\operatorname{Ham}}(\Sigma)$. The defect of $\hat{\mu}$ has upper bound $D(\hat{\mu}) \leq 2 D(\mu)$. If $\mu$ is stable, then so is $\hat{\mu}$, and if $\mu$ also has the Calabi property then the normalized version of $\hat{\mu}$

$$
\bar{\mu}: \widetilde{\operatorname{Ham}}(\Sigma) \rightarrow \mathbb{R} \quad \text { defined by } \quad \bar{\mu}(\varphi)=\zeta\left(\theta_{\epsilon}\right)^{-1} \mu\left(\phi_{\Theta_{\epsilon}(F)}\right)
$$

will also have the Calabi property, where $\zeta$ is the symplectic quasi-state associated to $\mu$.

The condition on $\epsilon$ is that the complement of $F_{\mathcal{P}}\left(\left\{e \in E_{\Sigma} \mid r(e) \leq 1-\epsilon\right\}\right)$ is a subset of the neighborhood $U$ of $\Delta_{\mathcal{P}}$. Since $\mu$ is the Calabi homomorphism on $\widetilde{\operatorname{Ham}}(M)_{U}$, it follows from (1.4) that $\zeta$ vanishes on functions supported in $U$. Therefore $M \backslash U$ has full quasi-measure $\tau_{\zeta}(M \backslash U)=1$, and hence $\zeta\left(\theta_{\epsilon}\right)>0$ since $\theta_{\epsilon}$ is strictly positive on $M \backslash U$. The map $\Theta$ can also be used to pullback symplectic quasi-states $\zeta$ on $M$ to $\Sigma$.

Theorem 1.4. Suppose that $\zeta: C^{\infty}(M) \rightarrow \mathbb{R}$ is a symplectic quasi-state and let $\theta$ as in (1.7) be such that $\zeta(\theta)>0$. Then the functional $\bar{\zeta}_{\theta}: C^{\infty}(\Sigma) \rightarrow \mathbb{R}$, defined as

$$
\bar{\zeta}_{\theta}(F)=\frac{\zeta(\Theta(F))}{\zeta(\theta)}
$$

is a symplectic quasi-state. The properties of vanishing on functions with displaceable support and being Ham invariant descend to $\bar{\zeta}_{\theta}$ should $\zeta$ have them. If $\zeta$ satisfies (1.5), then $\bar{\zeta}_{\theta}$ does as well with $C\left(\bar{\zeta}_{\theta}\right) \leq \frac{C(\zeta)}{\zeta(\theta)}\left\|\frac{\theta(r)}{\sqrt{1-r^{2}}}\right\|$.

Such $\theta$ exist if and only if the associated symplectic quasi-measure $\tau_{\zeta}\left(\Delta_{\mathcal{P}}\right)<1$, and in these cases they will exist with abundance. Theorems 1.3 and 1.4 are proved in Section 3.4, and examples of when they apply are given in Sections 2.2 and 2.3.

Remark 1.1. If Theorem 1.3 applies to a stable $\mu: \widetilde{\operatorname{Ham}}(M) \rightarrow \mathbb{R}$, then we have two ways to build symplectic quasi-states on $\Sigma$. We can reduce $\mu$ to $\bar{\mu}$ as 
in Theorem 1.3, and then form the associated quasi-state $\zeta_{\bar{\mu}}$. Alternatively, we can first form the associated quasi-state $\zeta=\zeta_{\mu}$ on $M$, and then reduce it to $\bar{\zeta}_{\theta_{\epsilon}}$ as in Theorem 1.4, where $\epsilon$ must be small enough for Theorem 1.3 to apply. These two symplectic quasi-states $\zeta_{\bar{\mu}}$ and $\bar{\zeta}_{\theta_{\epsilon}}$ are equal, which can be verified by checking on normalized Hamiltonians $F \in C^{\infty}(\Sigma)$

$$
\zeta_{\bar{\mu}}(F)=-\frac{\bar{\mu}\left(\phi_{F}\right)}{\operatorname{Vol}(\Sigma)}=-\frac{\mu\left(\phi_{\Theta_{\epsilon}(F)}\right)}{\zeta\left(\theta_{\epsilon}\right) \operatorname{Vol}(\Sigma)}=\frac{\zeta\left(\Theta_{\epsilon}(F)\right)}{\zeta\left(\theta_{\epsilon}\right)}=\bar{\zeta}_{\theta_{\epsilon}}(F) .
$$

Remark 1.2. Theorems 1.3 and 1.4 only rely on the Biran decomposition (1.6), for which the condition that $(M, \Omega, J)$ be Kähler is not essential. Let $\left(M^{2 n}, \Omega\right)$ be a closed symplectic manifold with $[\Omega] \in H^{2}(M ; \mathbb{Z})$ and let $\Sigma^{2 n-2}$ be a closed symplectic submanifold Poincaré dual to $k[\Omega]$ for $k \in \mathbb{N}$. In this setting $(M, \Omega ; \Sigma)$, Opshtein proved a Biran decomposition result $[$ Ops09, Theorem 1] where the skeleton $\Delta$ in (1.6) has zero volume. Under the additional assumption that $M \backslash \Sigma$ is a Weinstein manifold, $\Sigma$ is called a symplectic hyperplane section and for these Biran and Khanevsky [BK11, Section 2.3] explain that Biran's decomposition holds with the skeleton $\Delta$ being an isotropic cellular subspace. Closed symplectic submanifolds $\Sigma^{2 n-2} \subset\left(M^{2 n}, \Omega\right)$ that are Poincaré dual to $k[\Omega] \in H^{2}(M ; \mathbb{Z})$ exist for sufficiently large $k \in \mathbb{N}$ by Donaldson [Don96]. For $k$ sufficiently large, these Donaldson symplectic hypersurfaces also satisfy the condition that $M \backslash \Sigma$ is Weinstein by Giroux [Gir02, Proposition 11].

Remark 1.3. Suppose that in Theorem 1.3 we replaced Biran's symplectomorphism $F_{\mathcal{P}}$ in (1.6) with a symplectic embedding of a disk bundle over $\Sigma$ into a tubular neighborhood of $\Sigma$ in $M$. For a small enough tubular neighborhood of $\Sigma$, reducing any spectral quasi-morphism $\mu(a, \cdot)$ this way will result in the trivial quasi-morphism by Corollary 2.1. Therefore a priori Theorems 1.3 and 1.4 cannot be made into local constructions.

In [Bor11], the constructions in this paper are adjusted to the case of symplectic reduction for Hamiltonian $\mathbb{T}^{k}$-actions on a level set of the moment map $\Phi:(M, \omega) \rightarrow \mathbb{R}^{k}$ with full quasi-measure. In contrast to this paper, the constructions in [Bor11] are truly local in nature.

Remark 1.4. This method of reducing quasi-morphisms and symplectic quasi-states also works in the setting of Liouville domains $(M, \omega, L)$, when the Reeb vector field for $\alpha=\left.\left(\iota_{L} \omega\right)\right|_{\partial M}$ induces a free $S^{1}$-action on $\partial M$. Then the analogues of Theorems 1.3 and 1.4 hold and allow one to reduce quasimorphism and symplectic quasi-states on $M$ to the reduction $\Sigma=\partial M / S^{1}$.

\section{Remarks and examples}

2.1. A criterion for a spectral quasi-morphism to restrict to the Calabi homomorphism. From the definitions, whenever a stable homogeneous quasi-morphism on $\widetilde{\operatorname{Ham}}(M)$ restricts to the Calabi homomorphism 
on $\widetilde{\operatorname{Ham}}_{U}(M)$ for an open set $U$, the associated quasi-measure vanishes on $U$. It turns out that the converse is true as well for spectral quasi-morphisms.

Proposition 2.1. Let $\mu(a, \cdot): \widetilde{\operatorname{Ham}}(M) \rightarrow \mathbb{R}$ be a spectral quasi-morphism and let $\tau(a, \cdot)$ be its associated symplectic quasi-measure. If an open subset $U \subset M$ has zero measure $\tau(a, U)=0$, then $\mu$ restricts to the Calabi homomorphism on $\widetilde{\operatorname{Ham}}_{U}(M)$.

Proof. If $\varphi \in \widetilde{\operatorname{Ham}}_{U}(M)$, then it is generated by $F: M \times[0,1] \rightarrow \mathbb{R}$ such that $\operatorname{supp}\left(F_{t}\right) \subset U$ for all $t$. The fact that $\tau(a, U)=0$ implies that $\zeta(a, H)=0$ for all $H \in C^{\infty}(M)$ are zero outside of $U$. There exist functions $A, B: M \rightarrow \mathbb{R}$ that are zero outside $U$ so that as time-dependent functions $k A \leq F^{\# k} \leq k B$ and hence by the monotonicity of spectral invariants

$$
0=\zeta(A)=\lim _{k \rightarrow \infty} \frac{c(a, k A)}{k} \leq \lim _{k \rightarrow \infty} \frac{c\left(a, F^{\# k}\right)}{k} \leq \lim _{k \rightarrow \infty} \frac{c(a, k B)}{k}=\zeta(B)=0 .
$$

Therefore the limit term vanishes in (1.2) and hence $\mu(a, \varphi)=\operatorname{Cal}_{U}(\varphi)$.

We can now prove Theorem 1.1.

Proof of Theorem 1.1. By Theorem 1.2, a stably displaceable set $U$ has zero measure and hence by Proposition $2.1, \mu(a, \cdot)$ restricts to the Calabi homomorphism on $\widetilde{\operatorname{Ham}}_{U}(M)$.

A corollary to Theorem 1.1 is the following.

Corollary 2.1. Let $\Sigma \subset(M, \omega)$ be a closed nowhere coisotropic submanifold, then $\Sigma$ is stably displaceable and hence its has a neighborhood on which $\mu(a, \cdot)$ restricts to the Calabi homomorphism. A special case being when $\Sigma \subset M$ is a closed symplectic submanifold.

Proof. Observe that $\Sigma \times S^{1} \subset M \times T^{*} S^{1}$ is nowhere coisotropic and has at least one nonvanishing section of its normal bundle. Therefore by Gürel's displacement principle [Gür08, Theorem 1.1], we know that $\Sigma \times S^{1}$ is displaceable in $M \times T^{*} S^{1}$.

2.2. Reduction for subcritical polarizations. The skeleton $\Delta_{\mathcal{P}}$ of a polarization $\mathcal{P}=\left(M^{2 n}, \Omega, J ; \Sigma\right)$ is an isotropic cellular subspace and can be replaced with an isotropic $\mathrm{CW}$-complex $\Delta$ of the same dimension such that the decomposition (1.6) still holds [Bir01, Theorem 2.6.C]. A polarization is subcritical if $\operatorname{dim} \Delta_{\mathcal{P}}<n$ and is critical if $\operatorname{dim} \Delta_{\mathcal{P}}=n$, which are the only options since $\Delta_{\mathcal{P}}$ is isotropic. In the subcritical case, the isotopy theorem [BC01, Theorem 6.1.1] states that $\Delta$ is displaceable. The following is now a corollary of Theorems 1.3 and 1.4. 
Corollary 2.2. Let $\mathcal{P}=\left(M^{2 n}, \Omega, J ; \Sigma\right)$ be a subcritical polarization. Calabi quasi-morphisms $\mu: \widetilde{\operatorname{Ham}}(M) \rightarrow \mathbb{R}$ descend to $\bar{\mu}: \widetilde{\operatorname{Ham}}(\Sigma) \rightarrow \mathbb{R}$ as in Theorem 1.3. Symplectic quasi-states $\zeta$ on $(M, \omega)$ with the vanishing property descend to symplectic quasi-states $\bar{\zeta}$ on $\left(\Sigma, \Omega_{\Sigma}\right)$ as in Theorem 1.4 .

2.2.1. The Hirzebruch surfaces are hypersurfaces in subcritical polarizations. The Hirzebruch surfaces are given by

$$
\Sigma_{k}=\left\{(z, w) \in \mathbb{C P}^{2} \times \mathbb{C P}^{1} \mid z_{0} w_{0}^{k}+z_{1} w_{1}^{k}=0\right\},
$$

for $k \in \mathbb{N}$, when $k$ is even $\Sigma_{k}$ is diffeomorphic to $\mathbb{C P}^{1} \times \mathbb{C P}^{1}$, and when $k$ is odd $\Sigma_{k}$ is diffeomorphic to $\mathbb{C P}^{2}$ blown-up at $[0: 0: 1]$. Let $\omega_{n}$ be the Fubini-study form on $\mathbb{C P}^{n}$ such that $\int_{\mathbb{C P}^{1}} \omega_{n}=1$. Observe that the Poincaré dual of $\left[\Sigma_{k}\right] \in H_{4}\left(\mathbb{C P}^{2} \times \mathbb{C P}^{1} ; \mathbb{Z}\right)$ is $\left[\Omega_{k}\right]:=\left[\omega_{2}\right]+k\left[\omega_{1}\right]$. Therefore if $J$ is the standard complex structure, then $\mathcal{P}_{k}=\left(\mathbb{C P}^{2} \times \mathbb{C P}^{1}, \Omega_{k}, J ; \Sigma_{k}\right)$ is a polarization of degree 1 .

The holomorphic line bundle $\mathcal{L}_{k} \rightarrow \mathbb{C P}^{2} \times \mathbb{C P}^{1}$ determined by $\Sigma_{k}$, has an unique (up to scaling) holomorphic section $s_{k}$ with zero set $\Sigma_{k}$. Picking a hermitian metric $\|\cdot\|$ on $\mathcal{L}_{k}$ gives a function $\rho_{k}: \mathbb{C P}^{2} \times \mathbb{C P}^{1} \backslash \Sigma_{k} \rightarrow \mathbb{R}$ where

$$
\rho_{k}(z, w)=-\left\|s_{k}(z, w)\right\|^{2}=-\frac{\left|z_{0} w_{0}^{k}+z_{1} w_{1}^{k}\right|^{2}}{\|z\|^{2}\|w\|^{2 k}} .
$$

The skeleton $\Delta_{k}=\Delta_{\mathcal{P}_{k}}$ is defined to be the union of the stable manifolds for $\nabla \rho_{k}$. We have that

$$
\Delta_{k}=\left\{\left[\bar{w}_{0}^{k}: \bar{w}_{1}^{k}: 0\right] \times\left[w_{0}: w_{1}\right] \mid w \in \mathbb{C P}^{1}\right\}
$$

which is two-dimensional, and therefore the polarization $\mathcal{P}_{k}$ is subcritical. See [Bir01, Section 3] for similar examples. To summarize:

Proposition 2.2. The polarizations $\mathcal{P}_{k}=\left(\mathbb{C P}^{2} \times \mathbb{C P}^{1}, \Omega_{k}=\omega_{2}+k \omega_{1}, J ; \Sigma_{k}\right)$ given by the Hizebruch surfaces $\Sigma_{k}$ are subcritical and the symplectomorphism types of the hypersurfaces are as follows:

(1) $\left(\Sigma_{2 l},\left.\Omega_{2 l}\right|_{\Sigma_{2 l}}\right)$ is symplectomorphic to $\left(\mathbb{C P}^{1} \times \mathbb{C P}^{1}, \omega_{1} \oplus 3 l \omega_{1}\right)$,

(2) $\left(\Sigma_{2 l+1},\left.\Omega_{2 l+1}\right|_{\Sigma_{2 l+1}}\right)$ is symplectomorphic to $\left(\mathbb{C P}^{2} \# \overline{\mathbb{C P}^{2}}, \omega\right)$, where $\omega$ is the symplectic form with $\omega(L)=3 l+2$ for $L$ the general line and $\omega(E)=3 l+1$ for $E$ the exceptional divisor.

These two families of rational ruled surfaces have quasi-morphisms and symplectic quasi-states that descend from $\left(\mathbb{C P}^{2} \times \mathbb{C P}^{1}, \Omega_{k}\right)$, as in Corollary 2.2 .

Proof. The $\Sigma_{k}$ are ruled surfaces, since projection onto the $\mathbb{C P}^{1}$ factor $\pi_{k}: \Sigma_{k} \rightarrow \mathbb{C P}^{1}$ gives a $\mathbb{C P}^{1}$-fiber bundle. Hence by Lalonde and McDuff [LM96], the symplectomorphism type of $\left(\Sigma_{k}, \Omega_{k} \mid \Sigma_{k}\right)$ is determined by the cohomology class $\left[\left.\Omega_{k}\right|_{\Sigma_{k}}\right] \in H^{2}\left(\Sigma_{k}, \mathbb{Z}\right)$. A basis for $H_{2}\left(\Sigma_{k}, \mathbb{Z}\right)$ is given by

$$
F_{k}=\left[\pi_{k}^{-1}([1: b])\right]=\left[\left\{z_{0}+z_{1} b^{k}=0\right\} \times[1: b]\right] \quad \text { and } \quad D_{k}=\left[[0: 0: 1] \times \mathbb{C P}^{1}\right] \text {, }
$$


where $\Omega_{k}\left(F_{k}\right)=1$ and $\Omega_{k}\left(D_{k}\right)=k$, and the intersections are $F_{k} \cdot F_{k}=0$, $F_{k} \cdot D_{k}=1$, and $D_{k} \cdot D_{k}=-k$.

When $k=2 l$ is even, $\left(\Sigma_{2 l},\left.\Omega_{2 l}\right|_{\Sigma_{2 l}}\right)$ and $\left(\mathbb{C P}^{1} \times \mathbb{C P}^{1}, \omega\right)$ are symplectomorphic and the homology classes of the spheres are identified with

$$
\left[\mathbb{C P}^{1} \times p t\right]=F_{2 l} \quad \text { and } \quad\left[p t \times \mathbb{C P}^{1}\right]=l F_{2 l}+D_{2 l} .
$$

Therefore $\omega\left(\left[\mathbb{C P}^{1} \times p t\right]\right)=1$ and $\omega\left(\left[p t \times \mathbb{C P}^{1}\right]\right)=3 l$, which proves $(1)$.

When $k=2 l+1$ is odd, $\left(\Sigma_{2 l+1},\left.\Omega_{2 l}\right|_{\Sigma_{2 l}}\right)$ and $\left(\mathbb{C P}^{2} \# \overline{\mathbb{C P}^{2}}, \omega\right)$ are symplectomorphic and the homology classes of $L$ and $E$ are identified with

$$
L=(l+1) F_{2 l+1}+D_{2 l+1} \text { and } E=l F_{2 l+1}+D_{2 l+1} .
$$

Therefore $\omega(L)=3 l+2$ and $\omega(E)=3 l+1$, which proves $(2)$.

2.2.2. Subcritical polarizations from algebraic geometry. Biran and Jerby [BJ11] have proved that if $M \subset \mathbb{C P}^{m}$ is a smooth algebraic manifold whose dual variety $M^{*} \subset \mathbb{C P}^{m *}$ has codimension at least 2 , then a hyperplane section $\Sigma$ in $M$ gives a subcritical polarization $(M, \Sigma)$. Examples of such $M$ are the complex Grassmannians $\operatorname{Gr}(2,2 n+1)$ and $\mathbb{C P}^{n}$-bundles over smooth projective varieties of dimension less than $n$ with linear fibers. Refer to [Tev03, Section 9.2.C] for other examples.

2.3. Reduction for critical polarizations. If $\mathcal{P}=\left(M^{2 n}, \Omega, J ; \Sigma, \Delta_{\mathcal{P}}\right)$ is a critical polarization, then $\Delta_{\mathcal{P}}$ has dimension equal to $n$ and is possibly nondisplaceable. Therefore it is not enough that a quasi-morphism have the Calabi property for it to descend, however, in light of Proposition 2.1 we can say the following:

Corollary 2.3. Let $\mathcal{P}=\left(M^{2 n}, \omega, J ; \Sigma, \Delta_{\mathcal{P}}\right)$ be a critical polarization. Spectral quasi-morphisms $\mu: \widetilde{\operatorname{Ham}}(M) \rightarrow \mathbb{R}$ descend to $\bar{\mu}: \widetilde{\operatorname{Ham}}(\Sigma) \rightarrow \mathbb{R}$ as in Theorem 1.3, provided $\Delta_{\mathcal{P}}$ has a neighborhood $U$ with zero measure $\tau_{\mu}(U)=0$.

Example 2.1. Let $\Sigma$ be the diagonal in $\left(\mathbb{C P}^{1} \times \mathbb{C P}^{1}, \omega_{1} \oplus \omega_{1}\right)$. This forms a critical polarization and the anti-diagonal $\Delta$ is the corresponding skeleton [Bir01, Section 3.2.1]. The quantum homology $\mathrm{QH}_{4}\left(\mathbb{C P}^{1} \times \mathbb{C P}^{1}\right)$ splits into a direct sum of fields, with idempotents $a_{ \pm}$and corresponding spectral quasimorphisms $\mu_{ \pm}$, quasi-states $\zeta_{ \pm}$, and quasi-measures $\tau_{ \pm}$.

In [EP10a, Theorem 1.1] Eliashberg and Polterovich compute that $\Delta$ has a neighborhood $U$ with zero measure $\tau_{+}(U)=0$, in particular, $\tau_{+}(K)=1$ where $K$ is an exotic torus disjoint from $\Delta$. Therefore by Corollary 2.3, $\mu_{+}$and $\zeta_{+}$descend to $\left(\Sigma, \omega_{1} \oplus \omega_{1}\right)=\left(\mathbb{C P}^{1}, 2 \omega_{1}\right)$. The reduced quasi-state $\bar{\zeta}_{+}$is the median quasi-state, for it is the unique symplectic quasi-state that vanishes on displaceable sets [EP06, Section 8], but the uniqueness of $\mu\left(\left[S^{2}\right], \cdot\right)$ as a Calabi quasi-morphism on $\operatorname{Ham}\left(S^{2}\right)$ is unknown. 


\section{Proofs}

3.1. The general set-up. Let $(P, \xi)$ be a closed contact manifold with contact form $\alpha$ and assume the Reeb vector field $R$ generates a free $S^{1}$ action on $P$. The quotient $\Sigma=P / S^{1}$, has a symplectic form $\sigma=-\pi_{*} d \alpha$ induced by the projection $\pi: P \rightarrow \Sigma$. From this principal $S^{1}$-bundle, we can build the standard symplectic disk bundle $\pi:(E, \omega) \rightarrow(\Sigma, \sigma)$ modeled on $P$. The total space $E=P \times_{S^{1}} \mathbb{D}^{2}$ has a symplectic form given by

$$
\omega=\pi^{*} \sigma+d\left(r^{2} \alpha\right)=-d\left(\left(1-r^{2}\right) \alpha\right),
$$

where $r$ is the radial coordinate on the open unit disk $\mathbb{D}^{2}$. Note that $\operatorname{sgrad} r^{2}=R$, each fiber has area one (by taking $S^{1}=\mathbb{R} / \mathbb{Z}$ ), and $(\Sigma, \sigma)$ embeds symplectically into $E$ as the zero section. Given a polarization $\mathcal{P}=(M, \Omega, J ; \Sigma)$, the unit normal bundle $P \rightarrow \Sigma$ is a prequantization space for $\left(\Sigma, \Omega_{\Sigma}\right)$, and hence we can form the standard symplectic disk bundle $\left(E_{\Sigma}, \omega_{\text {can }}\right) \rightarrow\left(\Sigma, \Omega_{\Sigma}\right)$. This is the bundle from Biran's decomposition theorem (1.6), compare to [Bir01, Section 2] and [Ops09, Section 1].

Returning to the standard symplectic disk bundle $\pi:(E, \omega) \rightarrow(\Sigma, \sigma)$ modeled on $P$, let $\theta:[0,1] \rightarrow \mathbb{R}$ be nonnegative, zero in a neighborhood of one, and such that $\theta(r): E \rightarrow \mathbb{R}$ as a function of the radial coordinate $r$ is smooth. We have an associated linear, order preserving map

$$
\Theta: C^{\infty}(\Sigma, \sigma) \rightarrow C^{\infty}(E, \omega) \text { defined as } H \mapsto \theta \cdot \pi^{*} H \text {. }
$$

By integrating over the fiber, one can check that

$$
-\int_{0}^{1} \theta^{\prime}(r)\left(1-r^{2}\right)^{n} d r \int_{\Sigma} H \sigma^{n-1}=\int_{E} \Theta(H) \omega^{n},
$$

so $\Theta$ preserves the property of a function having zero mean and hence induces a map $\Theta: \mathcal{P} \mathcal{H}(\Sigma) \rightarrow \mathcal{P} \mathcal{H}(E)$. At times we will be interested in the maps $\Theta_{\epsilon}$ which are induced by $\theta_{\epsilon}(r):[0,1] \rightarrow \mathbb{R}$, which smoothly interpolates between $1-r^{2}$ when $r \leq 1-\epsilon$ and zero when $r \geq 1-\frac{\epsilon}{2}$.

For ease of notation and future applicability, will prove our lemmas in this general setting. Note that the $\Theta$ from (1.7) in Theorems 1.3 and 1.4 is just $\Theta$ from (3.1) combined with the use of the symplectomorphism $F_{\mathcal{P}}$ from (1.6) to view $\left(E_{\Sigma}, \omega_{\text {can }}\right)=(E, \omega)$ as an open subset of $M$. So our lemmas will be applicable to the setting of the theorems.

3.2. An overview of the proofs of Theorems 1.3 and 1.4. Before diving into the details of proving Theorems 1.3 and 1.4, let us step back and identify the main issue. We have a homogeneous quasi-morphism $\mu: \widetilde{\operatorname{Ham}}(E) \rightarrow \mathbb{R}$ and a map $\Theta: \mathcal{P} \operatorname{Ham}(\Sigma) \rightarrow \mathcal{P} \operatorname{Ham}(E)$, and we want to form a quasi-morphism $\hat{\mu}: \widetilde{\operatorname{Ham}}(\Sigma) \rightarrow \mathbb{R}$. Our situation can be summarized 
by the diagram:

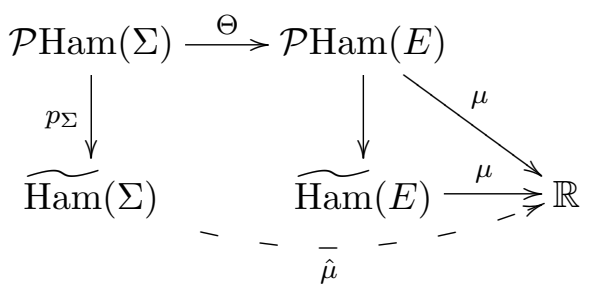

If $\Theta: \mathcal{H}(\Sigma) \rightarrow \mathcal{H}(E)$ preserved the Poisson brackets, then we would be done for $\Theta: \mathcal{P} \operatorname{Ham}(\Sigma) \rightarrow \mathcal{P} \operatorname{Ham}(E)$ would be a homomorphism and would descend to a homomorphism $\widehat{\Theta}: \widetilde{\operatorname{Ham}}(\Sigma) \rightarrow \widetilde{\operatorname{Ham}}(E)$, which we could use to pullback $\mu$ to a quasi-morphism on $\widetilde{\operatorname{Ham}}(\Sigma)$.

Unfortunately, $\Theta$ does not preserve the Poisson brackets, but since we only want a quasi-morphism, the full strength of a homomorphism is not necessary. In Section 3.3, we will formulate when a homogeneous quasimorphism $\mu: G \rightarrow \mathbb{R}$ can be pulled back by a map $H \rightarrow G$ (Lemma 3.1), and when $\mu$ will descend along a quotient $G \rightarrow H$ (Lemma 3.2). We will then show that $\mu: \mathcal{P} \operatorname{Ham}(E) \rightarrow \mathbb{R}$ pulls back along $\Theta_{\epsilon}$ to a homogeneous quasi-morphism $\Theta_{\epsilon}^{*} \mu: \mathcal{P} \operatorname{Ham}(\Sigma) \rightarrow \mathbb{R}$, which then descends along $p_{\Sigma}$ to a homogeneous quasi-morphism $\hat{\mu}: \widehat{\operatorname{Ham}}(\Sigma) \rightarrow \mathbb{R}$. The technical heart of these two steps occupies Lemmas 3.3 and 3.5, from which the other parts of Theorems 1.3 and 1.4 follow.

3.3. Two group theory lemmas. For these two lemmas, $G$ and $H$ will be groups and $\mu: G \rightarrow \mathbb{R}$ will be a homogeneous quasi-morphism with defect $D(\mu)$.

Definition 3.1. A map $\varphi: H \rightarrow G$ is $\mu$-quasi-homomorphism if there is some $D(\varphi, \mu)$ such that

$$
\left|\mu\left(\varphi\left(h_{1} h_{2}\right)^{-1} \varphi\left(h_{1}\right) \varphi\left(h_{2}\right)\right)\right| \leq D(\varphi, \mu) \quad \text { for all } h_{1}, h_{2} \in H .
$$

$\varphi$ is $\mu$-homogeneous if there is some $C$ such that

$$
\left|\mu\left(\varphi\left(h^{n}\right)^{-1} \varphi(h)^{n}\right)\right| \leq C \text { for all } n \in \mathbb{Z} \text { and } h \in H .
$$

Lemma 3.1. If $\varphi: H \rightarrow G$ is a $\mu$-quasi-homomorphism, then $\varphi$ pulls $\mu$ back to $\varphi^{*} \mu: H \rightarrow \mathbb{R}$, a quasi-morphism on $H$, with defect at most $D(\varphi, \mu)+2 D(\mu)$. If $\varphi$ is also $\mu$-homogeneous, then $\varphi^{*} \mu$ is a homogeneous quasi-morphism.

Proof. We have that

$$
\begin{aligned}
\left|\mu\left(\varphi\left(h_{1} h_{2}\right)\right)-\mu\left(\varphi\left(h_{1}\right)\right)-\mu\left(\varphi\left(h_{2}\right)\right)\right| & \leq\left|-\mu\left(\varphi\left(h_{1} h_{2}\right)\right)+\mu\left(\varphi\left(h_{1}\right) \varphi\left(h_{2}\right)\right)\right|+D(\mu) \\
& \leq\left|\mu\left(\varphi\left(h_{1} h_{2}\right)^{-1} \varphi\left(h_{1}\right) \varphi\left(h_{2}\right)\right)\right|+2 D(\mu) \\
& \leq D(\varphi, \mu)+2 D(\mu),
\end{aligned}
$$


so $\varphi^{*} \mu$ is a quasi-morphism. If $\varphi$ is $\mu$-homogeneous, then

$$
\begin{aligned}
\left|\mu\left(\varphi\left(h^{n}\right)\right)-\mu\left(\varphi(h)^{n}\right)\right| & =\left|\mu\left(\varphi\left(h^{n}\right)^{-1}\right)+\mu\left(\varphi(h)^{n}\right)\right| \\
& \leq\left|\mu\left(\varphi\left(h^{n}\right)^{-1} \varphi(h)^{n}\right)\right|+D(\mu) \leq C+D(\mu) .
\end{aligned}
$$

Dividing through by $n$ and taking the limit, we see that $\varphi^{*} \mu$ is its own homogenization.

Lemma 3.2. Let $\varphi: G \rightarrow H$ be a surjective homomorphism such that $\mu$ is bounded on the kernel of $\varphi$. Then $\varphi$ pushes $\mu$ forward to a homogeneous quasi-morphism

$$
\varphi_{*} \mu: H \rightarrow \mathbb{R} \text { where }\left(\varphi_{*} \mu\right)(h)=\mu(g) \quad \text { for any } g \in \varphi^{-1}(h),
$$

with defect $D(\mu)$.

Proof. The statement is clear provided that $\varphi_{*} \mu$ is well defined. Let $B \geq 0$ be such that $|\mu(\operatorname{ker} \varphi)| \leq B$. Now if $g_{1}$ and $g_{2}$ both map to $h \in H$, then $g_{1}^{n}$ and $g_{2}^{n}$ differ by an element of the kernel, so

$$
n\left|\mu\left(g_{1}\right)-\mu\left(g_{2}\right)\right|=\left|\mu\left(g_{1}^{n}\right)-\mu\left(g_{2}^{n}\right)\right| \leq B+D(\mu) .
$$

Now take the limit as $n$ goes to infinity, to get $\mu\left(g_{1}\right)=\mu\left(g_{2}\right)$.

3.4. The proofs of Theorems 1.3 and 1.4. We will need the following four lemmas, which are proved in Section 3.5.

Lemma 3.3. The map $\Theta: C^{\infty}(\Sigma, \sigma) \rightarrow C^{\infty}(E, \omega)$ preserves the vanishing of Poisson brackets, namely for $H, K \in C^{\infty}(\Sigma)$ we have that

$$
\{\Theta(H), \Theta(K)\}_{\omega}=\theta(r)^{2}\left\{\pi^{*} H, \pi^{*} K\right\}_{\omega}=\frac{\theta(r)}{1-r^{2}} \Theta\left(\{H, K\}_{\sigma}\right) .
$$

If $\left\{f_{t}\right\} \in \mathcal{P} \operatorname{Ham}(\Sigma)$ and $\left\{\widetilde{f}_{t}\right\} \in \mathcal{P} \operatorname{Ham}(E)$ are generated by $F \in \mathcal{P} \mathcal{H}(\Sigma)$ and $\Theta_{\epsilon}(F) \in \mathcal{P H}(E)$, then

$$
f_{t} \circ \pi=\pi \circ \widetilde{f}_{t} \text { when } r \leq 1-\epsilon .
$$

The term measuring the failure of $\Theta_{\epsilon}: \mathcal{P H}(\Sigma) \rightarrow \mathcal{P H}(E)$ to be a homomorphism

$$
\overline{\Theta_{\epsilon}(F \# G)} \#\left(\Theta_{\epsilon}(F) \# \Theta_{\epsilon}(G)\right): E \times[0,1] \rightarrow \mathbb{R}
$$

vanishes at points in $E$ with $r \leq 1-\epsilon$. This also holds for larger products as well, in particular for $\overline{\Theta_{\epsilon}\left(F^{\# k}\right)} \#\left(\Theta_{\epsilon}(F)^{\# k}\right)$.

Lemma 3.4. If $X \subset \Sigma$ is displaceable, then $\widetilde{X}_{a}=\pi^{-1}(X) \cap\{r \leq a\} \subset E$ is displaceable if $a<1$.

Lemma 3.5. Suppose that $F \in \mathcal{P H}(\Sigma)$ generates a loop $f$, which is a null homotopic $[f]=\mathbb{1}$ in $\widetilde{\operatorname{Ham}}(\Sigma)$, and let $\Theta_{\epsilon}(F) \in \mathcal{P H}(E)$ generate the path 
$\widetilde{f}$. Then as an element of $\widetilde{\operatorname{Ham}}(E),[\widetilde{f}]=[\eta]$ where $\eta$ is generated by a normalized Hamiltonian on $E$ that vanishes when $r \leq 1-\epsilon$.

Lemma 3.6. Let $\mu: \widetilde{\operatorname{Ham}}(M) \rightarrow \mathbb{R}$ be a stable homogeneous quasi-morphism and let $H \in C^{\infty}(M)$ be a normalized Hamiltonian. For any smooth $\lambda:[0,1] \rightarrow \mathbb{R}$,

$$
\mu\left(\phi_{\lambda H}\right)=\left(\int_{0}^{1} \lambda(t) d t\right) \mu\left(\phi_{H}\right)
$$

where $\lambda H \in \mathcal{P H}(M)$ is a time dependent normalized Hamiltonian.

\subsubsection{The proof of Theorem 1.3.}

Proof that $\mu$ descends to $\hat{\mu}$. By picking $\epsilon_{0}$ small, we can take the neighborhood $U$ of $\Delta_{\mathcal{P}}$ to be the complement of $F_{\mathcal{P}}\left(r \leq 1-\epsilon_{0}\right) \subset M$, and we will work with $\epsilon<\epsilon_{0}$.

If $F, G \in \mathcal{P H}(\Sigma)$ are normalized Hamiltonians, then by (3.5) in Lemma 3.3

$$
\overline{\Theta_{\epsilon}(F \# G)} \#\left(\Theta_{\epsilon}(F) \# \Theta_{\epsilon}(G)\right) \text { and } \overline{\Theta_{\epsilon}\left(F^{\# k}\right)} \# \Theta_{\epsilon}(F)^{\# k}
$$

are normalized and supported on $U$. Since $\mu$ restricted to $\widetilde{\operatorname{Ham}}_{U}(M)$ is the Calabi homomorphism, $\mu$ vanishes on (3.6) since they are normalized. Therefore by Lemma 3.1, $\Theta_{\epsilon}$ pulls back $\mu$ to a homogeneous quasi-morphism $\Theta_{\epsilon}^{*} \mu$ on $\mathcal{P H}(\Sigma)$ with defect at most $2 D(\mu)$. It is independent of $\epsilon$ since if $\epsilon^{\prime}, \epsilon<\epsilon_{0}$, then $\mu$ vanishes on $\overline{\Theta_{\epsilon}(F)} \# \Theta_{\epsilon^{\prime}}(F)$, for it is normalized and supported on $U$. Hence $\Theta_{\epsilon}^{*} \mu=\Theta_{\epsilon^{\prime}}^{*} \mu$, for they are homogeneous quasi-morphism a bounded distance apart.

If $F \in \mathcal{P H}(\Sigma)$ generates a null-homotopic element in $\widetilde{\operatorname{Ham}}(\Sigma)$, then by Lemma $3.5 \Theta_{\epsilon}(F)$ generates an element $\eta \in \widetilde{\operatorname{Ham}}(M)$, which can also be generated by a normalized Hamiltonian in $\mathcal{P H}(M)$ that is supported in $U$. Therefore by the Calabi property again, $\mu(\eta)=0$, and hence the quasimorphism $\Theta_{\epsilon}^{*} \mu$ vanishes on the kernel of $\mathcal{P} \mathcal{H}(\Sigma) \rightarrow \widetilde{\operatorname{Ham}}(\Sigma)$. Therefore by Lemma $3.2, \Theta_{\epsilon}^{*} \mu$ descends to $\widetilde{\operatorname{Ham}}(\Sigma)$ as the homogeneous quasi-morphism $\hat{\mu}$ in (1.8) with defect at most $2 D(\mu)$.

Proof that $\bar{\mu}$ inherits the stability and Calabi properties from $\mu$. Let $\mu$ be stable and have the Calabi property, and recall that $\bar{\mu}:=\zeta\left(\theta_{\epsilon}\right)^{-1} \hat{\mu}$. Observe that for normalized functions $H, K: \Sigma \rightarrow \mathbb{R}$,

$$
\min _{\Sigma}(H-K)=\min _{M}\left(\Theta_{\epsilon}(H)-\Theta_{\epsilon}(K)\right)
$$

and likewise for max. Hence the stability of $\bar{\mu}$ will follow from the stability of $\mu$. The normalization constant $\zeta\left(\theta_{\epsilon}\right)^{-1}$ is independent of $\epsilon$, for small $\epsilon$, since $\theta_{\epsilon}$ and $\theta_{\epsilon^{\prime}}$ Poisson commute implies $\zeta\left(\theta_{\epsilon}\right)-\zeta\left(\theta_{\epsilon^{\prime}}\right)=\zeta\left(\theta_{\epsilon}-\theta_{\epsilon^{\prime}}\right)=0$, for the difference is supported on the set $U$ where $\mu$ restricts to the Calabi homomorphism. 
To prove the Calabi property for $\bar{\mu}$, let $F: \Sigma \times[0,1] \rightarrow \mathbb{R}$ be supported on a displaceable set $V \subset \Sigma$ and let us denote $\operatorname{Vol}=\operatorname{Vol}(M)=\operatorname{Vol}(\Sigma)$. Consider the normalization terms for $F$ and $\Theta_{\epsilon}(F)$ :

$$
\lambda(t)=\mathrm{Vol}^{-1} \int_{\Sigma} F_{t} \Omega_{\Sigma}^{n-1} \text { and } \eta(t)=\mathrm{Vol}^{-1} \int_{M} \Theta_{\epsilon}\left(F_{t}\right) \Omega^{n}=C_{\epsilon} \lambda(t),
$$

where the last equality uses (3.2) and the notation $C_{\epsilon}=\mathrm{Vol}^{-1} \int_{M} \theta_{\epsilon} \Omega^{n}$. Since $\Theta_{\epsilon}(\lambda)-\eta=\lambda \cdot\left(\theta_{\epsilon}-C_{\epsilon}\right)$, by Lemma 3.6 we have that

$$
\mu\left(\phi_{\Theta_{\epsilon}(\lambda)-\eta}\right)=\left(\int_{0}^{1} \lambda(t) d t\right) \mu\left(\phi_{\theta_{\epsilon}-C_{\epsilon}}\right)=\mathrm{Cal}_{V}(F) \frac{\mu\left(\phi_{\theta_{\epsilon}-C_{\epsilon}}\right)}{\mathrm{Vol}} .
$$

By Lemma 3.4 the support of $\Theta_{\epsilon}(F)$ is displaceable, so therefore

$$
\mu\left(\phi_{\Theta_{\epsilon}(F)-\eta}\right)=\int_{0}^{1} \int_{M} \Theta_{\epsilon}\left(F_{t}\right) \Omega^{n} d t=C_{\epsilon} \operatorname{Cal}_{V}(F)=\frac{\int_{M} \theta_{\epsilon} \Omega^{n}}{\operatorname{Vol}} \operatorname{Cal}_{V}(F)
$$

Bringing this all together and using that $\Theta_{\epsilon}(F)$ and $\Theta_{\epsilon}(\lambda)$ commute gives

$$
\begin{aligned}
\bar{\mu}\left(\phi_{F-\lambda}\right) & =\zeta\left(\theta_{\epsilon}\right)^{-1}\left(\mu\left(\phi_{\Theta_{\epsilon}(F-\lambda)}\right)+\mu\left(\phi_{\Theta_{\epsilon}(\lambda)-\eta}\right)-\mu\left(\phi_{\Theta_{\epsilon}(\lambda)-\eta}\right)\right) \\
& =\zeta\left(\theta_{\epsilon}\right)^{-1}\left(\mu\left(\phi_{\Theta_{\epsilon}(F)-\eta}\right)-\mu\left(\phi_{\Theta_{\epsilon}(\lambda)-\eta}\right)\right) \\
& =\operatorname{Cal}_{V}(F) \zeta\left(\theta_{\epsilon}\right)^{-1} \frac{\int_{M} \theta_{\epsilon} \Omega^{n}-\mu\left(\phi_{\theta_{\epsilon}-C_{\epsilon}}\right)}{\operatorname{Vol}}=\operatorname{Cal}_{V}(F),
\end{aligned}
$$

as desired.

\subsubsection{The proof of Theorem 1.4 .}

Proof that $\zeta$ descends to $\bar{\zeta}_{\theta}$. The monotonicity and normalization of $\bar{\zeta}_{\theta}$ are immediate. As for quasi-linearity, if $F, G \in C^{\infty}(\Sigma)$ Poisson commute, then by (3.3) we have that $\Theta(F), \Theta(G) \in C^{\infty}(M)$ Poisson commute as well. Therefore

$$
\bar{\zeta}_{\theta}(F+G)=\frac{\zeta(\Theta(F)+\Theta(G))}{\zeta(\theta)}=\frac{\zeta(\Theta(F))+\zeta(\Theta(G))}{\zeta(\theta)}=\bar{\zeta}_{\theta}(F)+\bar{\zeta}_{\theta}(G),
$$

so we have that $\bar{\zeta}_{\rho}$ is in fact a symplectic quasi-state.

Proof that $\bar{\zeta}_{\theta}$ inherits properties from $\zeta$. Suppose that $\zeta$ vanishes on functions with displaceable support. If $H \in C^{\infty}(\Sigma)$ has displaceable support $X \subset \Sigma$, then by Lemma 3.4, $\Theta(H): M \rightarrow \mathbb{R}$ does as well and hence $\bar{\zeta}_{\theta}(H)=\zeta(\Theta(H))=0$.

Suppose that $\zeta: C^{\infty}(M) \rightarrow \mathbb{R}$ is $\operatorname{Ham}(M)$ invariant. Let $\left\{f_{t}\right\}$ be a Hamiltonian isotopy generated by $F \in \mathcal{P} \mathcal{H}(\Sigma)$. Let $\epsilon$ be small enough so that $\theta(r)$ vanishes when $r>1-\epsilon$, and let $\Theta_{\epsilon}(F) \in \mathcal{P} \operatorname{Ham}(M)$ generate the Hamiltonian isotopy $\left\{\widetilde{f}_{t}\right\}$. On the image of $F_{\mathcal{P}}$ in $M$, by (3.4) we have that $f_{t} \circ \pi \circ F_{\mathcal{P}}^{-1}=\pi \circ F_{\mathcal{P}}^{-1} \circ \widetilde{f}_{t}$, and hence for $H \in C^{\infty}(\Sigma)$ we have that

$$
\Theta\left(H \circ f_{t}\right)=\theta \cdot\left(H \circ f_{t} \circ \pi^{*} \circ F_{\mathcal{P}}^{-1}\right)=\left(\theta \cdot\left(H \circ \pi^{*} \circ F_{\mathcal{P}}^{-1}\right)\right) \circ \widetilde{f}_{t}=\Theta(H) \circ \widetilde{f}_{t} .
$$


Therefore $\bar{\zeta}_{\theta}$ is $\operatorname{Ham}(\Sigma)$ invariant, for

$$
\bar{\zeta}_{\theta}\left(H \circ f_{t}\right)=\frac{\zeta\left(\Theta\left(H \circ f_{t}\right)\right)}{\zeta(\Theta(1))}=\frac{\zeta\left(\Theta(H) \circ \widetilde{f}_{t}\right)}{\zeta(\Theta(1))}=\bar{\zeta}_{\theta}(H) .
$$

Finally, for the inequality (1.5) suppose that $\zeta$ satisfies it with $C(\zeta)$. Then by (3.3), for $H, K \in C^{\infty}(\Sigma)$ we have that

$$
\begin{aligned}
\Pi_{\bar{\zeta}_{\theta}}(H, K) & =\zeta(\theta)^{-1} \Pi_{\zeta}(\Theta(H), \Theta(K)) \leq \frac{C(\zeta)}{\zeta(\theta)} \sqrt{\|\{\Theta(H), \Theta(K)\}\|} \\
& \leq \frac{C(\zeta)}{\zeta(\theta)}\left\|\frac{\theta(r)}{\sqrt{1-r^{2}}}\right\| \sqrt{\|\{H, K\}\|} .
\end{aligned}
$$

So therefore $C\left(\bar{\zeta}_{\theta}\right) \leq \frac{C(\zeta)}{\zeta(\theta)}\left\|\frac{\theta(r)}{\sqrt{1-r^{2}}}\right\|$.

\subsection{The proofs of Lemmas 3.3, 3.4, 3.5, and 3.6.}

Proof of Lemma 3.3. We will start by computing $\pi_{*}\left(\operatorname{sgrad} \pi^{*} H\right)$. For $X \in$ $T_{(p, r)} E$ a vector,

$$
\begin{array}{rlr}
-d H\left(\pi_{*} X\right) & =\omega\left(\operatorname{sgrad} \pi^{*} H, X\right) & \\
& =\left(\pi^{*} \sigma+r^{2} d \alpha\right)\left(\operatorname{sgrad} \pi^{*} H, X\right) & \text { by } \iota_{\text {sgrad }} \pi^{*} H \\
& =\left(1-r^{2}\right) \sigma\left(\pi_{*}\left(\operatorname{sgrad} \pi^{*} H\right), \pi_{*} X\right) & \text { by } \sigma=-\pi_{*} d \alpha
\end{array}
$$

so we conclude that $\pi_{*}\left(\operatorname{sgrad} \pi^{*} H\right)=\frac{1}{1-r^{2}} \operatorname{sgrad} H$. Therefore

$$
\pi_{*}(\operatorname{sgrad} \Theta(H))=\theta(r) \pi_{*}\left(\operatorname{sgrad} \pi^{*} H\right)=\frac{\theta(r)}{1-r^{2}} \operatorname{sgrad} H .
$$

Now since $\pi^{*} H$ and $\theta(r)$ Poisson commute, the computation for (3.3) is the following:

$$
\begin{aligned}
\{\Theta(H), \Theta(K)\}_{\omega} & =\theta(r)^{2}\left\{\pi^{*} H, \pi^{*} K\right\}_{\omega}=\theta(r)^{2} d H\left(\pi_{*} \operatorname{sgrad} \pi^{*} K\right) \\
& =\frac{\theta(r)^{2}}{1-r^{2}} \pi^{*}\left(\{H, K\}_{\sigma}\right)=\frac{\theta(r)}{1-r^{2}} \Theta\left(\{H, K\}_{\sigma}\right) .
\end{aligned}
$$

Going to the path space side, using (3.7) we see that $\pi \circ \widetilde{f}_{t}: E \rightarrow \Sigma$ satisfies the differential equation

$$
\partial_{t}\left(\pi \circ \widetilde{f}_{t}\right)=\left(\frac{\theta_{\epsilon}(r)}{1-r^{2}} \operatorname{sgrad} F_{t}\right)_{\pi \circ \tilde{f}_{t}} \quad \text { with initial condition } \pi \circ \widetilde{f}_{0}=\pi \text {. }
$$

Staring at a point with $\theta_{\epsilon}(r)=1-r^{2}$, then $f_{t} \circ \pi$ also satisfies this equation, so (3.4) follows by the uniqueness of solutions to ODEs. Denoting by $\widetilde{(f g)_{t}}$ the Hamiltonian path generated by $\Theta_{\epsilon}(F \# G)$ we have that

$$
\begin{aligned}
\overline{\Theta_{\epsilon}(F \# G)} \#\left(\Theta_{\epsilon}(F) \# \Theta_{\epsilon}(G)\right) & =\left(-\Theta_{\epsilon}(F \# G)+\Theta_{\epsilon}(F) \# \Theta_{\epsilon}(G)\right) \circ \widetilde{(f g)_{t}} \\
& =\theta_{\epsilon}(r)\left(-G \circ f_{t}^{-1} \circ \pi+G \circ \pi \circ \widetilde{f}_{t}^{-1}\right) \circ \widetilde{(f g)_{t}},
\end{aligned}
$$


which vanishes when $r \leq 1-\epsilon$ since then we can use that $f_{t}^{-1} \circ \pi=\pi \circ \widetilde{f}_{t}^{-1}$. The proof for larger products is the same.

Proof of Lemma 3.4. Let $F \in \mathcal{P H}(\Sigma)$ generate the isotopy $f$ which displaces $X$. Pick $\epsilon>0$ so that $1-\epsilon>a$ and let $\tilde{f}$ be the isotopy generated by $\Theta_{\epsilon}(F) \in \mathcal{P H}(E)$. It follows from (3.4) that $\widetilde{f}$ displaces $\widetilde{X}_{a}$.

Proof of Lemma 3.5. Let $\varphi_{t}^{s}$ be a homotopy of loops in $\operatorname{Ham}(\Sigma)$ based at $\mathbb{1}$, between the loop $\left\{\varphi_{t}^{0}=f_{t}\right\}_{t \in[0,1]}$ and the constant loop $\left\{\varphi_{t}^{1}=\mathbb{1}\right\}_{t \in[0,1]}$. For $s$ fixed, let $F_{t}^{s}: \Sigma \rightarrow \mathbb{R}$ be the Hamiltonian in $\mathcal{P H}(\Sigma)$ generating the Hamiltonian loop $\left\{\varphi_{t}^{s}\right\}_{t \in[0,1]}$ in $\operatorname{Ham}(\Sigma)$, via

$$
\partial_{t} \varphi_{t}^{s}=\left(\operatorname{sgrad} F_{t}^{s}\right)_{\varphi_{t}^{s}} \quad \text { with } \quad \varphi_{0}^{s}=\mathbb{1} .
$$

Note that $F_{t}^{0}=F_{t}$ and $F_{t}^{1}=0$. While for $t$ fixed, let $G_{t}^{s}: \Sigma \rightarrow \mathbb{R}$ be the Hamiltonian in $\mathcal{P H}(\Sigma)$ generating the Hamiltonian path $\left\{\varphi_{t}^{s}\right\}_{s \in[0,1]}$ in $\operatorname{Ham}(\Sigma)$, via

$$
\partial_{s} \varphi_{t}^{s}=\left(\operatorname{sgrad} G_{t}^{s}\right)_{\varphi_{t}^{s}} \quad \text { with } \quad \varphi_{t}^{0}=f_{t}
$$

They are related by the equation

$$
\partial_{s} F_{t}^{s}=\partial_{t} G_{t}^{s}+\left\{F_{t}^{s}, G_{t}^{s}\right\} .
$$

Fixing $s$, the Hamiltonian $\Theta_{\epsilon}\left(F_{t}^{s}\right): E \rightarrow \mathbb{R}$ in $\mathcal{P} \mathcal{H}(E)$ will generate a Hamiltonian path $\left\{\psi_{t}^{s}\right\}_{t \in[0,1]}$ in $\operatorname{Ham}(E)$, via

$$
\partial_{t} \psi_{t}^{s}=\left(\operatorname{sgrad} \Theta_{\epsilon}\left(F_{t}^{s}\right)\right)_{\psi_{t}^{s}} \quad \text { with } \quad \psi_{0}^{s}=\mathbb{1} .
$$

As $s$ varies, $\psi_{s}^{t}$ will be a homotopy of Hamiltonian paths in $\operatorname{Ham}(E)$, between the paths $\left\{\psi_{t}^{0}=\widetilde{f}_{t}\right\}_{t \in[0,1]}$ and $\left\{\psi_{t}^{1}=\mathbb{1}\right\}_{t \in[0,1]}$. However, this will not be a homotopy of loops, since in particular $\left\{\widetilde{f}_{t}\right\}_{t \in[0,1]}$ may not be a loop. Letting $t$ be fixed, let $H_{t}^{s}: E \rightarrow \mathbb{R}$ be the Hamiltonian in $\mathcal{P H}(E)$ generating the Hamiltonian path $\left\{\psi_{t}^{s}\right\}_{s \in[0,1]}$ in $\operatorname{Ham}(E)$, via

$$
\partial_{s} \psi_{t}^{s}=\left(\operatorname{sgrad} H_{t}^{s}\right)_{\psi_{t}^{s}} \quad \text { with } \quad \psi_{t}^{0}=\widetilde{f}_{t} .
$$

Just as in (3.8), we have the relation

$$
\partial_{s} \Theta_{\epsilon}\left(F_{t}^{s}\right)=\partial_{t} H_{t}^{s}+\left\{\Theta_{\epsilon}\left(F_{t}^{s}\right), H_{t}^{s}\right\} .
$$

Define the path $\left\{\eta_{u}=\left(\psi_{1}^{u}\right)^{-1} \psi_{1}^{0}\right\}_{u \in[0,1]}$ in $\operatorname{Ham}(E)$, which is a path from $\eta_{0}=\mathbb{1}$ to $\eta_{1}=\psi_{1}^{0}$, using that $\psi_{t}^{1}=\mathbb{1}$. Observe that $\Psi_{t}^{s}=\psi_{t}^{s} \eta_{s t}$ is a homotopy of paths in $\operatorname{Ham}(E)$, between the paths

$$
\left\{\Psi_{t}^{0}=\psi_{t}^{0}=\tilde{f}_{t}\right\}_{t \in[0,1]} \quad \text { and } \quad\left\{\Psi_{t}^{1}=\eta_{t}\right\}_{t \in[0,1]} .
$$

Since $\Psi$ is a homotopy of paths with fixed endpoints,

$$
\Psi_{0}^{s}=\mathbb{1} \quad \text { and } \quad \Psi_{1}^{s}=\psi_{1}^{s} \eta_{s}=\psi_{1}^{0},
$$

we have proved that $[\widetilde{f}]=[\eta]$ in $\widetilde{\operatorname{Ham}}(E)$. 
The path $\eta$ is generated by the normalized Hamiltonian $\left(-H_{1}^{u} \circ \psi_{1}^{u}\right)$, which we claim vanish at points with $r \leq 1-\epsilon$. It suffices to prove this for $H_{1}^{u}$ since $\psi_{t}^{s}$ preserves the level sets of $r$. Applying $\Theta_{\epsilon}$ to (3.8) and using (3.3) of Lemma 3.3 gives that

$$
\partial_{s} \Theta_{\epsilon}\left(F_{t}^{s}\right)=\partial_{t} \Theta_{\epsilon}\left(G_{t}^{s}\right)+\frac{\left(1-r^{2}\right)}{\theta_{\epsilon}(r)}\left\{\Theta_{\epsilon}\left(F_{t}^{s}\right), \Theta_{\epsilon}\left(G_{t}^{s}\right)\right\} .
$$

When $r \leq 1-\epsilon$, we have that $\theta_{\epsilon}(r)=1-r^{2}$ and so the differential equations (3.9) and (3.10) agree. Therefore by the method of characteristics for PDE's, $H_{t}^{s}=\Theta_{\epsilon}\left(G_{t}^{s}\right)$ when $r \leq 1-\epsilon$. The fact that $\varphi$ is a homotopy of paths with fixed endpoints means that $G_{1}^{s}=0$, and therefore $H_{1}^{s}=0$ when $r \leq 1-\epsilon$.

Proof of Lemma 3.6. If $L(t)=\int_{0}^{t} \lambda(s) d s$ and $h_{t}$ is the flow generated by $\operatorname{sgrad}(H)$, then the Hamiltonian $\lambda H$ generates the Hamiltonian isotopy $\phi_{\lambda H}=\left\{h_{L(t)}\right\}_{t \in[0,1]}$. By a time reparameterization, in $\widetilde{\operatorname{Ham}}(M)$ we have that $\left[\left\{h_{L(t)}\right\}\right]=\left[\left\{h_{t L(1)}\right\}\right]$ and hence $\mu\left(\phi_{\lambda H}\right)=\mu\left(\phi_{L(1) H}\right)$. If $m$ is an integer, then $\mu\left(\phi_{m H}\right)=\mu\left(\phi_{H}^{m}\right)=m \mu\left(\phi_{H}\right)$ since $\mu$ is homogeneous and hence $\mu\left(\phi_{a H}\right)=a \mu\left(\phi_{H}\right)$ when $a$ is rational. Since $\mu$ is stable, this extends to any real scalar, so in particular $\mu\left(\phi_{L(1) H}\right)=L(1) \mu\left(\phi_{H}\right)$.

\subsection{The proof of Theorem 1.2 .}

Proof of Theorem 1.2. Since $X \times S^{1}$ is displaceable in $M \times T^{*} S^{1}$, it is also displaceable in $M \times S^{1} \times[-R, R]$ where the last coordinate is the vertical direction in $T^{*} S^{1}$. Capping $S^{1} \times[-R, R]$ off on the top and bottom with half spheres creates an sphere. Therefore by Moser we may assume that $X \times S^{1} \subset M \times\left(S^{2}, \Omega\right)$ is displaceable, where $\left(S^{2}, \Omega\right)$ is a round sphere in $\mathbb{R}^{3}$ with coordinates $(x, y, z), \Omega$ is the induced area form, and $S^{1}$ is the equator $\{z=0\} \subset S^{2}$.

Let $U$ and $V=\{|z|<2 \epsilon\}$ be open neighborhoods of $X$ and $S^{1}$ respectively such that $U \times V \subset M \times S^{2}$ is still displaceable. Let $\rho: M \rightarrow \mathbb{R}$ be a cutoff function with support in $U$ that is a constant 1 near $X$. On $S^{2}$, let $V_{1}=\{z>\epsilon\}$ and $V_{2}=\{z<-\epsilon\}$, so $\left\{V=V_{0}, V_{1}, V_{2}\right\}$ is an open cover of $S^{2}$ and let $\phi_{i}(z)$ be a Poisson commuting subordinate partition of unity. Note that each $U \times V_{i} \subset M \times S^{2}$ is displaceable.

Let $\pi_{1}: M \times S^{2} \rightarrow M$ and $\pi_{2}: M \times S^{2} \rightarrow S^{2}$ be the projections and let $\zeta\left(a \otimes\left[S^{2}\right], \cdot\right)$ be the partial symplectic quasi-state on $M \times S^{2}$ associated to the idempotent $a \otimes\left[S^{2}\right] \in \mathrm{QH}_{2 n+2}\left(M \times S^{2}\right)$, see [EP09, Section 3.5]. Since the $\phi_{i}$ pullback to a Poisson commuting partition on unity on $M \times S^{2}$,

$$
\zeta\left(a \otimes\left[S^{2}\right], \pi_{1}^{*} \rho\right)=\zeta\left(a \otimes\left[S^{2}\right], \sum_{i=0}^{2} \pi_{1}^{*} \rho \cdot \pi_{2}^{*} \phi_{i}\right)=0 .
$$


The above vanishes by the partial additivity and vanishing property $[\mathbf{E P 0 9}$, Theorem 3.6] of partial symplectic quasi-states, using that the $\pi_{1}^{*} \rho \cdot \pi_{2}^{*} \phi_{i}$ Poisson commute and have displaceable support. Finally, by the product formula for spectral invariants [EP09, Theorem 5.1], we have that

$$
\zeta(a, \rho)=\zeta\left(a \otimes\left[S^{2}\right], \pi_{1}^{*} \rho\right)=0 .
$$

It now follows that from the definition of $\tau(a)$ that $\tau(a, X)=0$.

\section{Questions}

We will end this paper with two questions regarding the process of passing from a quasi-morphism $\mu: \widetilde{\operatorname{Ham}}(M) \rightarrow \mathbb{R}$ to its reduction $\bar{\mu}: \widetilde{\operatorname{Ham}}(\Sigma) \rightarrow \mathbb{R}$ as described in Theorem 1.3.

(1) If $\mu$ descends from the universal cover to $\mu: \operatorname{Ham}(M) \rightarrow \mathbb{R}$, does its reduction also descend to $\bar{\mu}: \operatorname{Ham}(\Sigma) \rightarrow \mathbb{R}$ ?

(2) If $\mu=\mu(a, \cdot): \widetilde{\operatorname{Ham}}(M) \rightarrow \mathbb{R}$ is a spectral quasi-morphism for some idempotent $a \in \mathrm{QH}_{2 n}(M)$, then does its reduction $\bar{\mu}=\mu(\bar{a}, \cdot)$ come for some idempotent $\bar{a} \in \mathrm{QH}_{2 n-2}(\Sigma)$ ?

The trouble with proving $(1)$ is that $\Theta_{\epsilon}: \mathcal{P} \operatorname{Ham}(M) \rightarrow \mathcal{P} \operatorname{Ham}(\Sigma)$ does not preserve loops due to the discrepancy between $\theta_{\epsilon}(r)$ and $1-r^{2}$. For $(2)$ the trouble is that there is no natural candidate for $\bar{a}$ since there is no known natural map $\mathrm{QH}_{2 n}(M) \rightarrow \mathrm{QH}_{2 n-2}(\Sigma)$ preserving the ring structure.

\section{References}

[Aar91] J.F. Aarnes, Quasi-states and quasi-measures, Adv. Math. 86(1) (1991), 41-67.

[BC01] P. Biran and K. Cieliebak, Symplectic topology on subcritical manifolds, Comment. Math. Helv. 76(4) (2001), 712-753.

[BEP12] L. Buhovsky, M. Entov and L. Polterovich, Poisson brackets and symplectic invariants, Selecta Math. (N.S.), 18(1) (2012), 89-157.

[Bir01] P. Biran, Lagrangian barriers and symplectic embeddings, Geom. Funct. Anal. 11(3) (2001), 407-464.

[Bir06] P. Biran, Lagrangian non-intersections, Geom. Funct. Anal. 16(2) (2006), 279-326.

[BJ11] P. Biran and Y. Jerby, The symplectic topology of projective manifolds with small dual, 2011, arXiv:1107.0174v1.

[BK11] P. Biran and M. Khanevsky, A Floer-Gysin exact sequence for Lagrangian submanifolds, 2011, arXiv:1101.0946v1.

[Bor11] M.S. Borman, Quasi-states, quasi-morphisms, and the moment map, Int. Math. Res. Not. (to appear), 2011, arXiv:1105.1805v3.

[Buh10] L. Buhovsky, The 2/3-convergence rate for the Poisson bracket, Geom. Funct. Anal. 19(6) (2010), 1620-1649. 
[Cal09] D. Calegari, scl, MSJ Mem., 20, Math. Soc. Japan, Tokyo, 2009.

[CV08] F. Cardin and C. Viterbo, Commuting Hamiltonians and Hamilton-Jacobi multi-time equations, Duke Math. J. 144(2) (2008), 235-284.

[Don96] S. K. Donaldson, Symplectic submanifolds and almost-complex geometry, J. Differential Geom. 44(4) (1996), 666-705.

[EP03] M. Entov and L. Polterovich, Calabi quasimorphism and quantum homology, Int. Math. Res. Not. 2003(30) (2003), 1635-1676.

[EP06] M. Entov and L. Polterovich, Quasi-states and symplectic intersections, Comment. Math. Helv. 81(1) (2006), 75-99.

[EP08] M. Entov and L. Polterovich, Symplectic quasi-states and semi-simplicity of quantum homology, in 'Toric topology', Contemp. Math., 460, Amer. Math. Soc., Providence, RI, 2008, 47-70.

[EP09] M. Entov and L. Polterovich, Rigid subsets of symplectic manifolds, Compos. Math. 145(3) (2009), 773-826.

[EP10a] Y. Eliashberg and L. Polterovich, Symplectic quasi-states on the quadric surface and Lagrangian submanifolds, 2010, arXiv:1006.2501v1.

[EP10b] M. Entov and L. Polterovich, $C^{0}$-rigidity of Poisson brackets, in 'Symplectic topology and measure preserving dynamical systems', Contemp. Math., 512, 25-32, Amer. Math. Soc., Providence, RI, 2010.

[EPZ07] M. Entov, L. Polterovich and F. Zapolsky, Quasi-morphisms and the Poisson bracket, Pure Appl. Math. Q. 3(4, Special Issue: In honor of Grigory Margulis. Part 1) (2007), 1037-1055.

[FOOO11] K. Fukaya, Y.-G. Oh, H. Ohta and K. Ono, Spectral invariants with bulk quasimorphisms and Lagrangian Floer theory, 2011, arXiv:1105.5123v1.

[Gir02] E. Giroux, Géométrie de contact: de la dimension trois vers les dimensions supérieures, in Proceedings of the International Congress of Mathematicians, Vol. II (Beijing, 2002), Higher Ed. Press, Beijing, 2002, 405-414.

[Gür08] B. Gürel, Totally non-coisotropic displacement and its applications to Hamiltonian dynamics, Commun. Contemp. Math. 10(6) (2008), 1103-1128.

[Kot04] D. Kotschick, What is... a quasi-morphism? Notices Amer. Math. Soc. 51(2) (2004), 208-209.

[LM96] F. Lalonde and D. McDuff, The classification of ruled symplectic 4-manifolds, Math. Res. Lett. 3(6) (1996), 769-778.

[MS98] D. McDuff and D. Salamon, Introduction to symplectic topology, Oxford Mathematical Monographs, The Clarendon Press, Oxford University Press, New York, 2nd ed., 1998.

[Ops09] E. Opshtein, Polarizations and symplectic isotopies, 2009, arXiv:0911.3601v1.

[Ost06] Y. Ostrover, Calabi quasi-morphisms for some non-monotone symplectic manifolds, Algebr. Geom. Topol. 6 (2006), 405-434 (electronic).

[Pol01] L. Polterovich, The geometry of the group of symplectic diffeomorphisms, Lectures in Mathematics ETH Zürich, Birkhäuser Verlag, Basel, 2001.

[She11] E. Shelukhin, The action homomorphism, quasimorphisms and moment maps on the space of compatible almost complex structures, 2011, arXiv:1105.5814v1. 
[Tev03] E. A. Tevelev, Projectively dual varieties, J. Math. Sci. (N.Y.) 117(6) (2003), 4585-4732.

[Ush11] M. Usher, Deformed Hamiltonian Floer theory, capacity estimates, and Calabi quasimorphisms, Geom. Topol. 15(3) (2011), 1313-1417.

Department of Mathematics

UNIVERSITY OF CHICAGO

Chicago, Illinois 60637

E-mail address: borman@math.uchicago.edu

Received 08/10/2010, accepted 06/20/2011

I would like to thank my advisor Leonid Polterovich for his wonderful help, guidance, and encouragement related to this work. I am grateful to Paul Biran for his generous help with examples of subcritical polarizations and explaining his work with Yochay Jerby to me. I would also like to thank Egor Shelukhin for useful discussions. This work was partially supported by the NSF-grant DMS 1006610. 